\title{
Deep Sedation or General Anesthesia for ERCP?
}

\author{
Nirav Thosani $\cdot$ Subhas Banerjee
}

Received: 14 August 2013/Accepted: 15 August 2013/Published online: 29 August 2013

(C) Springer Science+Business Media New York 2013

Ideal sedation for endoscopic procedures should maximize patient comfort and safety. However, during many endoscopic procedures performed under conscious (i.e., moderate) sedation, patient comfort is compromised to some extent in the interest of safety. Although conscious sedation was the mainstay for most endoscopic procedures over the initial decades of endoscopy, anesthesia services have increasingly been utilized over recent years to provide deeper levels of sedation [1]. The key driver for increased anesthesia utilization over the last decade has been the need to improve patient comfort, satisfaction, and safety while simultaneously improving the efficiency of endoscopy units. A significant facilitating factor has been the introduction and availability of new pharmaceutical agents such as propofol, which allow rapid induction of deep sedation while also enabling rapid recovery [2]. Propofol sedation for gastroscopy and colonoscopy is as safe as sedation using traditional agents, whether administered by anesthesia providers or under the direction of endoscopists [3]. Numerous studies attest to its benefits, including improved patient cooperation during the procedure, improved patient satisfaction, reduced procedural and recovery times, and, consequently, improved throughput and efficiency of endoscopy units [4]. In 2007, approximately $25 \%$ of U.S. colonoscopies and gastroscopies were performed with anesthesiologist-assisted sedation [1]. Given the relative simplicity and brevity of many of these basic endoscopic procedures and the significant cost burden associated with the use of anesthesia, this practice remains

N. Thosani $\cdot$ S. Banerjee $(\bowtie)$

Division of Gastroenterology, Stanford University

School of Medicine, Stanford, CA, USA

e-mail: sbanerje@stanford.edu controversial and consequently has not been universally adopted.

In contrast, endoscopic retrograde cholangiopancreatography (ERCP) is a more complex endoscopic procedure that usually takes longer to perform. A recent study evaluating patients undergoing ERCP under conscious sedation found that between a third and a half of patients experienced pain and discomfort during the procedure and periprocedural period [5]. An additional study indicated that the procedural failure rate in patients undergoing ERCP with sedation was double the failure rate when general anesthesia was utilized (14 vs. $7 \%$ ). The higher failure rate with conscious sedation was entirely due to ERCPs that were terminated prematurely $(8.5 \%)$ due to inadequate sedation [6]. These data constitute powerful arguments for the use of deep propofol sedation or general anesthesia in patients undergoing ERCP and, indeed, this is the trend in many academic medical centers across the U.S. However, there is a dearth of literature evaluating the most appropriate type of sedation or anesthesia for complex endoscopic procedures such as ERCP. A recent Cochrane review identified only four randomized controlled studies comparing moderate sedation using midazolam and meperidine with propofol administered by non-anesthesiologists for ERCP. No difference in mortality, serious cardio-respiratory complications, or patient satisfaction between the two sedation techniques was noted, although patients receiving propofol sedation had a faster and better recovery profile [7].

From the viewpoint of endoscopy room efficiency, propofol sedation where safe is preferable to general anesthesia as it allows for a more rapid procedure room turnover. Anesthesia practices vary from center to center and also between anesthesiologists at the same center, with some anesthesiologists judiciously choosing between deep 
propofol sedation and general anesthesia/intubation based on procedural and patient characteristics, while others consistently intubate every ERCP patient. The latter approach is neither reasonable nor desirable, but persists for a variety of reasons. Firstly, there is a paucity of data available to guide anesthesiologists in selecting between deep propofol sedation and general anesthesia/intubation for ERCP. Moreover, providing anesthesia in remote locations, including endoscopy suites, may be challenging for the anesthesiologist, due to unfamiliarity with the environment and, potentially, a lack of immediate access to all the equipment, medications and support personnel they are accustomed to within the operating room [8]. Furthermore, older studies evaluating anesthesia provision at remote 'out of operating room (OR)' locations have indicated an increased risk of complications and death compared to anesthesia administered in the operating room [8], and this historical data may influence the mind-set, comfort level, and decision making of anesthesiologists assigned to work in the endoscopy unit. These studies pre-date the careful cardio-respiratory monitoring utilized today and also predominantly draw data from Emergency room and ICU settings, and should not therefore be generalized to the endoscopy unit [8]. Nevertheless, as a consequence of some or all of these factors, anesthesiologists assigned to the endoscopy unit may feel out of their element and may therefore be more likely to choose general anesthesia/ intubation over deep propofol sedation.

The timely study by Barnett et al. [9] published in this issue assesses the safety of anesthesia-directed deep sedation (ADDS) in non-intubated patients compared to general endotracheal anesthesia (GET) for ERCP. The investigators prospectively evaluated 438 patients undergoing ERCP over a 5-month study period, using a dedicated study instrument to record intra-procedure and post-procedure adverse events, including hypoxia, need for mask ventilation, unplanned endotracheal intubation, hypotension requiring use of vasopressors, cardiac arrhythmia requiring treatment, the use of reversal agents, and cardiac arrest [9]. Of 438 patients, $393(89.7 \%)$ received ADDS and only 45 $(10.2 \%)$ received GET. The GET group had a significantly higher mean body mass index and a greater number of American Society of Anesthesiologist (ASA) class 4 patients compared to the ADDS group. Both groups were similar with regards to patient age, co-morbidities, indications, and technical difficulty of ERCP. Intra-procedure events occurred in $25.7 \%$ of ADDS cases and $35.6 \%$ of GET cases, with no significant complications reported in either group. Sixteen $(3.7 \%)$ of the ADDS cases required conversion to GET anesthesia during the procedure with one ERCP performed with ADDS prematurely terminated due to an intra-procedure event. Of the 16 GET converted cases, a higher proportion had chronic obstructive pulmonary disease (25 vs. $10 \%$ ) than non-converted cases. Similarly, a higher proportion of converted cases were ASA class 4 (25 vs. $6.4 \%$ ) compared with all ADDS cases. Post-procedure recovery room events were uncommon in both groups. Overall, the data indicate that use of propofol deep sedation without intubation is feasible and safe for the majority of patients undergoing ERCP, especially nonobese, healthier patients [9].

The study undoubtedly has limitations which may affect its overall results, particularly the non-randomized study design which might have contributed to a possible selection bias towards general anesthesia in obese patients. Again, the familiarity of individual anesthesiologists with the endoscopy unit environment and their comfort level with ERCP may have driven choices between ADDS and GET. The authors indicate that all anesthesiologists participating in this study rotated regularly through the endoscopy unit. Anesthesiologists who provide sedation on a routine basis for ERCP have been shown to have lower overall anesthesia and procedure times, fewer complications, and a lower intubation rate compared to anesthesiologists who provide anesthesia on an 'ad hoc' basis for ERCP [10]. In addition, the study was conducted at a high-volume tertiary care endoscopy unit with highly skilled endoscopists and consequently a relatively short procedure time. Procedural duration is an independent predictor of respiratory and cardiovascular complications in patients undergoing endoscopy with propofol sedation [11]. Therefore these results might not be generalizable to low-volume units with less skilled endoscopists or to units where anesthesiologists provide anesthesia infrequently and on an ad hoc basis. Regardless, this important study has indicated that when skilled anesthesiologists and endoscopists work together, it is possible to perform $90 \%$ of ERCPs at a tertiary care endoscopy unit under deep propofol sedation safely and with a low conversion rate to general anesthesia/intubation.

Given the growing utilization of anesthesia for complex endoscopic procedures and the simultaneous increasing pressure on hospitals to improve efficiency, we need more studies comparing the safety, efficiency, and costs of deep propofol sedation and general anesthesia/intubation for patients undergoing advanced endoscopic procedures. Further prospective studies are also needed to evaluate the effect of experience and comfort of anesthesiologists with ERCP in determining their choice between deep propofol sedation and general anesthesia/intubation. Undoubtedly, some ERCP patients have co-morbidities that mandate general anesthesia with intubation. In addition, the complexity of ERCP is variable, with a complexity scale of 1-4 proposed by the Quality Committee of the American Society of Gastrointestinal Endoscopy [12]. Grade 1 and 2 procedures are considered technically less challenging and typically can be completed in a relatively short time 
compared to more complex (grade 3 and 4) ERCPs [12]. Unless patient characteristics dictate general anesthesia, it is our opinion that most grade 1 and 2 and many grade 3 ERCPs can be safely performed using deep propofol sedation without endotracheal intubation. In contrast, for more complex procedures, the patient's safety and tolerance over the course of the longer procedure may be a significant factor in determining procedural success and general anesthesia/intubation may be preferable. Thus, it is imperative that endoscopists discuss each ERCP with anesthesiologists in order to help guide decision making. It is important to communicate the expected complexity of the procedure and estimated time to perform it, together with any known gastrointestinal issues that might contribute to decision making such as increased aspiration risk related to known gastroparesis or gastric outlet obstruction.

Finally, there is a compelling need for anesthesia divisions to develop smaller, dedicated, 'out of OR' anesthesia teams to provide sedation/anesthesia outside the operating room. This approach would allow for a smaller group of anesthesiologists to gain significant familiarity and experience in the delivery of sedation/anesthesia outside the operating room, including the endoscopy unit. Ideally, endoscopists in collaboration with dedicated anesthesiologists should develop standardized protocols to determine optimal sedation strategies for ERCP based on the grade of complexity of the procedure as well as on patient characteristics. This may bring us closer to achieving the perfect balance of patient comfort, safety, and endoscopy unit efficiency.

\section{References}

1. Inadomi JM, Gunnarsson CL, Rizzo JA, Fang H. Projected increased growth rate of anesthesia professional-delivered sedation for colonoscopy and EGD in the United States: 2009 to 2015 Gastrointest Endosc. 2010;72:580-586.

2. Cohen LB, Wecsler JS, Gaetano JN, et al. Endoscopic sedation in the United States: results from a nationwide survey. Am J Gastroenterol.. 2006;101:967-974.

3. Rex DK, Deenadayalu VP, Eid E, et al. Endoscopist-directed administration of propofol: a worldwide safety experience. Gastroenterology.. 2009;137:1229-1237.

4. Wang D, Chen C, Chen J, et al. The use of propofol as a sedative agent in gastrointestinal endoscopy: a meta-analysis. PLOS ONE. . 2013;8:e53311.

5. Jeurnink SM, Steyerberg E, Kuipers E, Siersema P. The burden of endoscopic retrograde cholangiopancreatography (ERCP) performed with the patient under conscious sedation. Surg Endosc.. 2012;26:2213-2219.

6. Raymondos K, Panning B, Bachem I, Manns MP, Piepenbrock S, Meier PN. Evaluation of endoscopic retrograde cholangiopancreatography under conscious sedation and general anesthesia. Endoscopy.. 2002;34:721-726.

7. Garewal D, Powell S, Milan SJ, Nordmeyer J, Waikar P. Sedative techniques for endoscopic retrograde cholangiopancreatography Cochrane Database Syst Rev. 2012;6:CD007274.

8. Cook T, Behringer EC, Benger J. Airway management outside the operating room: hazardous and incompletely studied. Curr Opin Anaesthesiol.. 2012;25:461-469.

9. Barnett SR, Berzin T, Sankara S et al. Deep Sedation without intubation for ERCP is appropriate in healthy, non-obese patients. Dig Dis Sci (Epub ahead of print). doi:10.1007/s10620-013-2783-x.

10. Goudra BG, Singh PM, Sinha AC. Anesthesia for ERCP: impact of anesthesiologist's experience on outcome and cost. Anesthesiol Res Pract.. 2013;2013:570518.

11. Garewal D, Vele L, Waikar P. Anaesthetic considerations for endoscopic retrograde cholangio-pancreatography procedures. Curr Opin Anaesthesiol.. 2013;26:475-480.

12. Cotton PB, Eisen G, Romagnuolo J, et al. Grading the complexity of endoscopic procedures: results of an ASGE working party. Gastrointest Endosc. 2011;73:868-874. 